\title{
Lateral confinement of fast electrons and its impact on laser ion acceleration
}

\author{
N. Iwata $\odot,{ }^{1,2, *}$ A. J. Kemp, ${ }^{3}$ S. C. Wilks, ${ }^{3}$ K. Mima,${ }^{1,4}$ D. Mariscal,${ }^{3}$ T. Ma,${ }^{3}$ and Y. Sentoku $\odot^{1}$ \\ ${ }^{1}$ Institute of Laser Engineering, Osaka University, 2-6 Yamadaoka, Suita, Osaka 565-0871, Japan \\ ${ }^{2}$ Institute for Advanced Co-Creation Studies, 1-1 Yamadaoka, Suita, Osaka 565-0871, Japan \\ ${ }^{3}$ Lawrence Livermore National Laboratory, Livermore, California 94550, USA \\ ${ }^{4}$ Graduate School for the Creation of New Photon Industries, 1955-1 Kurematsu, Nishiku, Hamamatsu, Shizuoka 141-1201, Japan
}

(Received 30 January 2020; revised 20 August 2020; accepted 21 May 2021; published 9 June 2021)

\begin{abstract}
In intense laser-plasma interactions, maximizing the density of fast electrons in the laser spot area is key to achieving plasma heating and particle acceleration. We find that when the laser spot size is large compared with the target foil thickness, fast electrons circulating in the foil show a "random walk" in the lateral direction due to the scattering by fluctuating fields at the plasma surface inside the spot area. We model the lateral motion as a diffusion, and find the resulting diffusion velocity is much slower than the speed of the ballistic transport. Hence, fast electrons accumulate in the spot region, and over time their density becomes typically 10 times greater than the laser-accelerated fast electron density. The enhancement of fast electron density in the target pushes the ion acceleration to more efficient regime.
\end{abstract}

DOI: 10.1103/PhysRevResearch.3.023193

\section{INTRODUCTION}

High-power lasers with relativistic intensities $>10^{18} \mathrm{~W} / \mathrm{cm}^{2}$ are available around the world. Applications using relativistic laser-plasma interactions have been studied such as laser-driven ion acceleration [1,2], intense x-ray [3], neutron [4-6], and positron [7,8] generation, and fast ignition-based laser fusion [9,10]. For these applications, generation and confinement of high-energy density plasma is a key to gain high efficiency.

In laser interactions with overdense targets, fast electrons accelerated at the laser-irradiated front surface play a role in transferring energy from laser to plasma particles inside the target. The loss of fast electrons from the laser spot area due to their divergence in the lateral direction has been thus discussed as a crucial problem for the efficient energy coupling. Efforts to confine fast electrons in the interaction area have been made, e.g., applying external magnetic fields [11] and using mass limited target [12] or layered target composed of materials with different resistivity [13].

When the target is a thin foil, the fast electrons can get bounced back by the sheath electric potential at the target rear. This recirculation increases the effective number of fast electrons in the foil, affecting the sheath field generation [14] and resulting an enhanced target-normal-sheath-acceleration (TNSA) of ions [15]. For quasi-one-dimensional (1D) situations, fast electrons accumulate in foil thickness $L$ as the laser

\footnotetext{
*iwata-n@ile.osaka-u.ac.jp
}

Published by the American Physical Society under the terms of the Creative Commons Attribution 4.0 International license. Further distribution of this work must maintain attribution to the author(s) and the published article's title, journal citation, and DOI. pulse duration exceeds $2 L / c$ [16,17] where $c$ is the light speed.

Recently, kilojoule $(\mathrm{kJ})$ class lasers with relativistic intensities, over picosecond (ps) pulse durations, and large focal spots of several tens to $100 \mu \mathrm{m}$ have become available [18-21]. When such a $\mathrm{kJ} / \mathrm{ps}$ laser is irradiated onto a foil target, fast electrons with average energies beyond the ponderomotive scaling [22] have been observed in experiments $[23,24]$ and in simulations [25-31]. The increase of the average energy is associated with the interaction of fast electrons, which circulate in the expanding foil plasma, with the laser field multiple times in the over-ps pulse duration $[25,28]$. If the fast electrons recirculate for a longer time in the laser spot area, they have more chance to be exposed to the laser field to gain high energies. The increase of the average energy of fast electrons contributes in boosting the maximum proton energy in TNSA [23-26]. Therefore, confining the fast electrons in the spot area is crucial for the efficient ion acceleration.

In this paper, we report a lateral confinement of fast electrons in large spot laser and thin foil interactions. When the laser spot radius is sufficiently large compared with the foil thickness, the fast electrons exhibit a random walk in the lateral direction by experiencing multiple scattering by the fluctuating fields at the plasma surfaces in recirculation. The random walk works as a lateral confinement of the electrons, and results in an accumulation of fast electrons in the spot area. In Sec. II, using two-dimensional (2D) particle-in-cell (PIC) simulations, we show the random walk and the resulting accumulation of fast electrons in the farget. In Sec. III a theoretical model of the lateral confinement is presented. Based on the model, we derive the achievable accumulation density of fast electrons and compare it with the PIC results in Sec. IV. In Sec. V the impact of the accumulated fast electron density on ion acceleration is discussed. Conclusions are given in Sec. VI. 

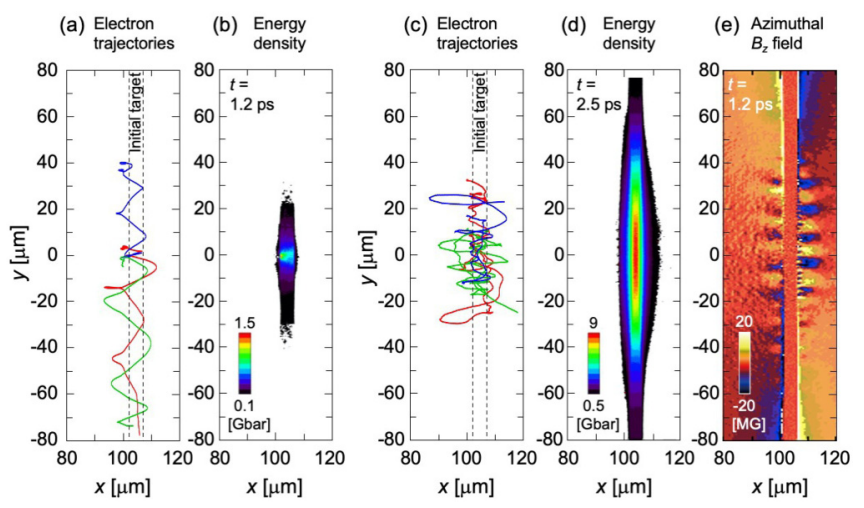

FIG. 1. 2D PIC simulations with laser spot radii $1.4 \mu \mathrm{m}$ (a), (b) and $35 \mu \mathrm{m}$ (c)-(e). Panels (a) and (c) are trajectories of fast electrons in the simulations, (b) and (d) are snapshots of the electron energy densities at $t=1.2 \mathrm{ps}$ and $t=2.5 \mathrm{ps}$, respectively, and (e) is the snapshot of the azimuthal magnetic field $B_{z}$ at $t=1.2 \mathrm{ps}$.

\section{LATERAL CONFINEMENT OF FAST ELECTRONS}

We consider the fast electron motion in interactions of a foil plasma with a relativistic laser light with the normalized amplitude $a_{0} \equiv e E_{L} / m_{e} c \omega_{L}>1$. Here $e, E_{L}, m_{e}, c$, and $\omega_{L}$ are elementary charge, laser electric field, electron rest mass, light speed, and laser frequency, respectively. Electrons at the front surface of the plasma are launched into the foil by the $\mathbf{v} \times \mathbf{B}$ force of the laser field. In the front and rear sides of the foil plasma, the fast electrons are exposed to quasistatic sheath electric and magnetic fields. The sheath electric field works as a trapping force in the target normal direction, while the surface magnetic field changes the angle of the electron trajectories. We show such interactions in Fig. 1 by using 2D PIC simulations with PICLS code [32] for a $5 \mu \mathrm{m}$-thick fully ionized deuteron foil plasma with density $n_{0}=100 n_{c}$ irradiated by a laser light with $a_{0}=1.4$ and wavelength $1 \mu \mathrm{m}$ where $n_{c}=m_{e} \omega_{L} /\left(4 \pi e^{2}\right)$ is the critical density. We use a small laser spot radius $w=1.4 \mu \mathrm{m}$ [Figs. 1(a) and 1(b)] and a large spot radius $w=35 \mu \mathrm{m}$ [Figs. 1(c)-1(e)] with a Gaussian lateral intensity profile. The laser amplitude rises within 100 fs with a Gaussian shape, and after that the amplitude is kept constant as $a_{0}$. The laser front reaches to the foil surface at $t=0.34 \mathrm{ps}$. The laser beam axis is in the $x$ direction, and the plasma is distributed uniformly in the $y$ direction. We set a $2 \mu \mathrm{m}$ pre-plasma in front of the foil whose density drops exponentially from $10 n_{c}$ with a scale length $0.5 \mu \mathrm{m}$. The system size is $200 \mu \mathrm{m}$ in the $x$ direction and $160 \mu \mathrm{m}$ in the $y$ direction. The mesh size is $20 \mathrm{~nm}$, and the particle number per cell (ppc) for each species is 20 . For particle trajectory tracking, we use the reduced simulations with 4 ppc for each species.

In Figs. 1(a) and 1(c), we show three trajectories of electrons picked up from the particles initially located at the critical density in the laser spot. For the small spot [Fig. 1(a)], during recirculation in the foil plasma, the electrons escape almost ballistically from the spot area. It is known that source electrons produced at the laser-plasma interface have a finite divergence angle. Filamentation of the laser beam at nearcritical density, electron acceleration in the modulated plasma,
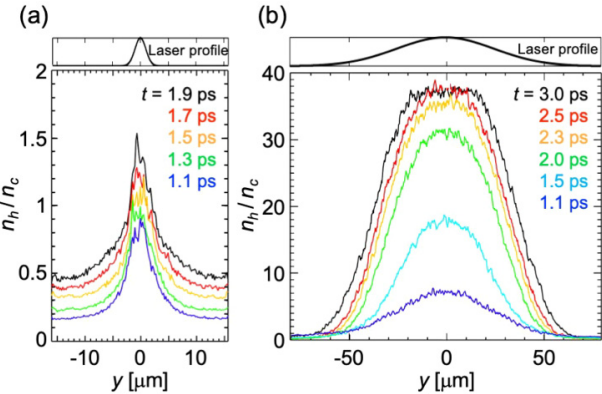

(c)

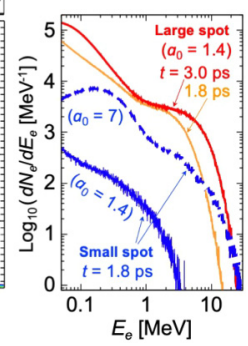

FIG. 2. Time evolutions of the lateral density profile of electrons having energies over $50 \mathrm{keV}$ with $a_{0}=1.4$ for (a) small $(w=1.4 \mu \mathrm{m})$ and (b) large $(w=35 \mu \mathrm{m})$ spot lasers. The incident laser profiles are shown in the upper panels. (c) Electron energy distributions in the spot area $|y|<w$ for the small (blue) and large (red and orange) spots with $a_{0}=1.4$, and for the small spot with $a_{0}=7.0$ (blue dashed).

and scattering of electrons by magnetic fields near the laserplasma interface result in a divergence angle of around $1 \mathrm{rad}$ for the source electrons [29]. In the large spot [Fig. 1(c)], the electrons exhibit random motions in the lateral $y$-direction and tend to stay in the spot area. This random motion originates from the interaction with the fluctuating fields at the modulated front and rear plasma surfaces as shown in Fig. 1(e), where the Weibel magnetic filaments grow in expanding plasmas $[33,34]$. The modulation scale length is about the laser wavelength $\lambda_{L}$ [35], and the condition $w \gg \lambda_{L}$ results the random scattering of the circulating electrons.

The achieved energy density of electrons in the large spot case reaches 9 Gbar, which is 18 times higher than that in the small spot case ( 0.5 Gbar) as in Figs. 1(b) and 1(d). This is due to the random walk in the large spot, by which the average flow velocity of the fast electrons becomes slower than the small spot case, resulting efficient confinement of fast electrons in the spot area. Here Figs. 1(b) and 1(d) are taken when the energy density inside the target at the laser axis $y=0$ reaches its maximum value.

Due to the transition from ballistic motion to random walk behavior, the fast electron density $n_{h}$ in the spot area inside the foil becomes much higher than the relativistic critical density. Figure 2 shows the time evolutions of $n_{h}$ for the [Fig. 2(a)] small and [Fig. 2(b)] large spot cases. The density profiles are taken at the center of the foil in the simulations. The lateral amplitude profiles of the incident lasers are shown in the upper panels. The fast electron density around the center $y=0$ in the large spot case [Fig. 2(b)] increases to $37 n_{c}$ at $t=2.5 \mathrm{ps}$ and saturates afterwards. This density is about 25 times higher than that reached in the small spot case [Fig. 2(a)], $\simeq 1.5 n_{c}$. Since the electrons accelerated into the foil have a finite divergence angle, the density $n_{h}$ in the small spot case (a) is smaller than the relativistic critical density especially in early time. Note that in Figs. 2(a) and 2(b), we take the density of electrons whose energies are higher than a threshold energy $50 \mathrm{keV}$ which is one fourth of the ponderomotive energy $T_{p}=(\gamma-1) m_{e} c^{2}$ where $\gamma=\left(1+a_{0}^{2} / 2\right)^{1 / 2}$ in the average relativistic factor [22]. For the Maxwellian energy distribution whose slope temperature is given by $T_{p}$, more than half of particles distribute above the threshold energy. The velocity 
corresponding to $50 \mathrm{keV}$ is about $40 \%$ of the light speed and is collisionless in a ps timescale. In the energy range $>50 \mathrm{keV}$, numerical heating is negligible.

Figure 2(c) presents the electron energy distributions in the spot area $|y|<w$. The blue solid line is the spectrum for the small spot case [Fig. 2(a)], and orange and red lines are those for the large spot case [Fig. 2(b)] during the accumulation $(t=$ $1.8 \mathrm{ps})$ and after the saturation $(t=3.0 \mathrm{ps})$, respectively. The number of fast electrons in the large spot is about 150 times larger than that in the small spot. This ratio 150 is much larger than the ratio of the spot size between the large spot and small spot cases, i.e., $35 \mu \mathrm{m} / 1.4 \mu \mathrm{m}=25$, indicating the effect of the lateral confinement. We also show the spectrum with the small spot but having higher amplitude $a_{0}=7.0$, which has the same laser power with the large spot case, by the blue dashed line. The number of over-MeV electrons for the $a_{0}=$ 7.0 case is an order of magnitude less than that for the large spot with $a_{0}=1.4$. Moreover, in the large spot case, the highenergy tail extends to more than $10 \mathrm{MeV}$, which is comparable to the $a_{0}=7.0$ case. The high-energy component in the large spot case corresponds to the superthermal electrons typically seen in the large spot $\mathrm{kJ}$ laser interactions $[23,25,28]$.

\section{THEORETICAL MODELING}

We model the fast electron accumulation in the large laser spot. When the circulating fast electrons come out to the modulated plasma surface, the electron motion is affected by the fluctuating surface magnetic and electric fields, and scattered randomly in the momentum space. We here model the scattering as a complete diffused reflection at the foil surfaces which results an isotropic angular distribution of the electrons in the momentum space. Such a random angular scattering can be described as a diffusion in the lateral direction [36]. We write the average step size in the lateral direction as $\Delta y=L \tan \bar{\theta}$, and the average period of the circulation as $\Delta t=L /(v \cos \bar{\theta})$, where $L$ is the length of the circulation in the longitudinal$x$ direction, $v$ is the velocity of the electron, and $\bar{\theta}$ is the average scattering angle of the electron at the foil surfaces. The length $L$ is approximately the same as the foil thickness for electrons with energy $T_{p}$ especially in early time of the interaction. Note that the surface magnetic field in Fig. 1(e) is about $10 \mathrm{MG}$ on average. The Larmor radius of an electron with the ponderomotive energy is about $0.2 \mu \mathrm{m}$, which is smaller than the Debye length at the relativistic critical density with the temperature $T_{p}$. Therefore, electrons can change the direction of motion by the cyclotron gyration during they reflux back to the foil in the sheath electric potential. We can thus consider the isotropic scattering condition. The average scattering angle is then given by

$$
\bar{\theta}=\frac{\int_{0}^{\pi / 2} \theta \sin \theta d \theta}{\int_{0}^{\pi / 2} \sin \theta d \theta}=1,
$$

which is about $57^{\circ}$.

The lateral flow velocity of the fast electrons for the random walk is derived as follows. If the fast electron motion in the lateral direction is ballistic, the lateral velocity is given as $u_{\text {bal }}=v \sin \bar{\theta}$. For the random walk case, we obtain the diffusion scale length $L_{\mathrm{dif}}$ by solving the diffusion equation

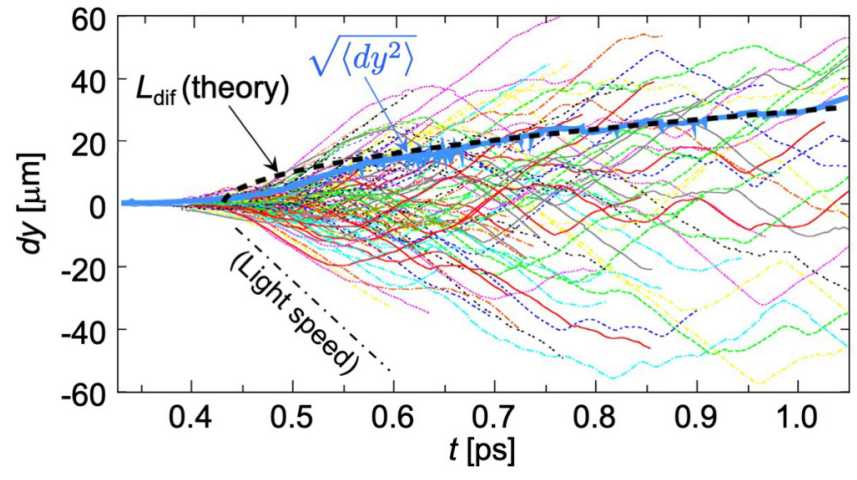

FIG. 3. Lateral deviation length of electrons tracked in the 2D PIC simulation for $a_{0}=1.4$ with a uniform laser profile in the lateral direction. The average deviation for the tracked electrons is shown by the blue bold line. The diffusion scale length $L_{\text {dif }}$ derived in Eq. (2) is presented by the black dashed line. A light speed trajectory is indicated by a black dash-dot line for reference.

$\partial P / \partial t=D \partial^{2} P / \partial y^{2}$. The probability for the electron to move from the center $(y=0)$ to the position $y$ is derived as $P \propto$ $\exp \left(-y^{2} / L_{\text {dif }}^{2}\right)$ with

$$
L_{\mathrm{dif}}=(2 D t)^{1 / 2}=\sqrt{L t u_{\mathrm{bal}} \tan \bar{\theta}},
$$

where $D=(\Delta y)^{2} /(2 \Delta t)$ is the diffusion coefficient.

The lateral velocity of the random walk electrons can be obtained from $u_{\mathrm{dif}}=d L_{\mathrm{dif}} / d t$. At the position of the spot radius, $L_{\mathrm{dif}}=w$, we have

$$
u_{\mathrm{dif}}=\frac{D}{w}=\frac{L}{w} \frac{\tan \bar{\theta}}{2} u_{\mathrm{bal}} .
$$

Equation (3) indicates that the lateral flow velocity becomes much slower for the random walk motion than the ballistic velocity, when the factor $(L \tan \bar{\theta}) / 2 w$ is less than order unity, i.e., $\Delta y / w \ll 1$. For instance, $u_{\text {dif }} \simeq 0.11 u_{\text {bal }}$ for $w=35 \mu \mathrm{m}$ and $L=5 \mu \mathrm{m}$. The stochastic nature of the circulating electrons in the large spot results in a slow lateral escape and thus an effective confinement in the lateral direction.

We confirm the above-derived diffusion velocity by using a 2D PIC simulation where the laser profile in the lateral $y$ direction is set to be uniform. Here a periodic boundary in the $y$ direction is used, while the other simulation parameters are the same as the large spot simulation in Fig. 2(b) $\left(a_{0}=1.4\right)$. In Fig. 3 we show the lateral deviation of electrons from the initial positions, $d y(t)=y(t)-y_{0}$. We selected 100 electrons from those located at the critical density in the pre-plasma region initially, and tracked their trajectories. Energies of the electrons change randomly in the recirculation process [25]. The tracking is continued until the particle energy exceeds $5 \mathrm{MeV}$, because the circulation period of such energetic electrons tends to be longer than a few hundred fs, and thus they do not represent the circulating electrons.

In early time in Fig. 3, all the tracked electrons move in the same trajectory, since all the electrons see the same plane wave and uniform sheath field. The electron trajectories begin to deviate each other after the surface modulation grows up in $100 \mathrm{fs}$, which is the timescale of the ion motion $2 \pi \omega_{\mathrm{pi}}^{-1}$ at the critical density where $\omega_{\mathrm{pi}}$ is the ion plasma frequency. 

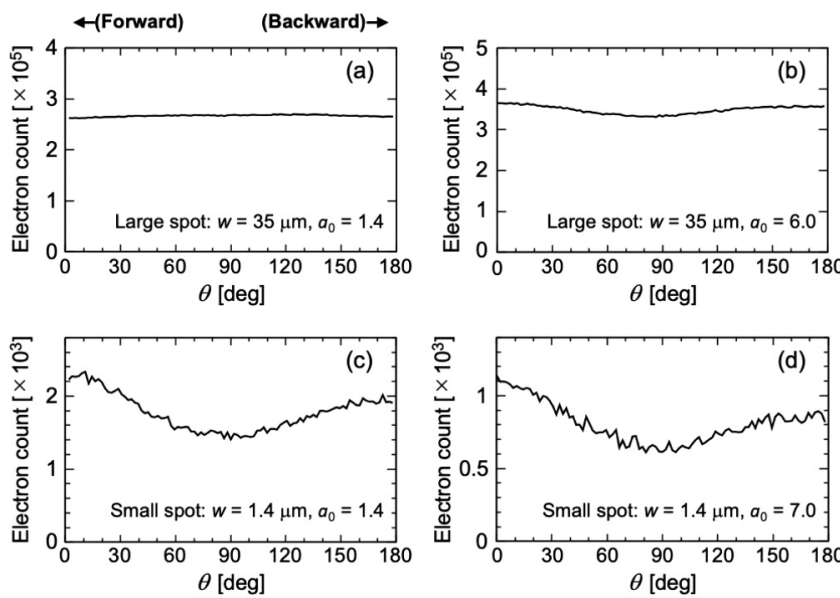

FIG. 4. Angular distribution of electrons whose energies are greater than about one fourth of the ponderomotive energy and less than $6 \mathrm{MeV}$ in the velocity space inside the foil in the spot area. Panels (a) and (b) are the distributions in the large spot cases ( $w=35 \mu \mathrm{m}$ ) for $a_{0}=1.4$ and (b) $a_{0}=6.0$, respectively, and (c) and (d) are the distributions in the small spot cases $(w=1.4 \mu \mathrm{m})$ for $a_{0}=1.4$ and $a_{0}=7.0$, respectively. The initial target thickness is $5 \mu \mathrm{m}$ for all the cases. $\theta=0$ corresponds to the forward (positive $x$ ) direction.

The blue bold line labeled as $\sqrt{\left\langle d y^{2}\right\rangle}$ is the lateral deviation averaged over the tracked electrons, which presents the statistical diffusion length from the original position. We also plot the diffusion scale length $L_{\text {dif }}$ given by Eq. (2) by the black dashed line. Here we set the initial position of $L_{\mathrm{dif}}$ as $y=0$ and $t=0.44$ ps at which the peak intensity of the laser reaches the front surface of the foil. The averaged lateral deviation obtained from the simulation is consistent with the theoretical estimation. We also see the diffusion velocity, which is the gradient of the averaged deviation line, is much slower than the light speed indicated by the black dash-dot line.

In Fig. 4 we show the angular distribution of fast electrons in the velocity space observed in the spot area inside the target in the PIC simulations for the large and small spot cases. $\theta=$ 0 corresponds to the forward (positive $x$ ) direction. Here we counted the electrons whose energies are greater than about one fourth of the ponderomotive energy and less than $6 \mathrm{MeV}$. The angular distribution for the large spot cases [Fig. 4(a)] for $a_{0}=1.4$ and [Fig. 4(b)] for $a_{0}=6.0$ are almost isotropic due to the random scattering, so that the average scattering angle is $\bar{\theta}=1 \mathrm{rad}$ as derived in Eq. (1). As the spot size decreases to less than the foil thickness as Figs. 4(c) and 4(d), the electrons show nonuniform angular distributions having peaks along the laser axis. For the small spot cases [Figs. 4(c) and 4(d)], the number of electrons directed to the forward direction is larger than that directed to the backward direction, suggesting a part of fast electrons escapes from the spot area before recirculate into the target.

\section{ACCUMULATION DENSITY OF FAST ELECTRONS}

We now estimate the achievable density of fast electrons by the lateral confinement by the random walk in the $2 \mathrm{D}$ con- figuration. We assume the density becomes a steady state as observed in the PIC simulation in Fig. 2(b). The steady-state density $n_{h}$ is determined by the balance between the number of electrons escape from the spot area and that injected from the laser-plasma interface as

$$
0=D \frac{\partial^{2} n_{h}}{\partial y^{2}}+S
$$

where $S=\gamma n_{c} v / L$ is the source term indicating the inflow of the fast electrons from the interface. We assume that the laser has a uniform amplitude profile in the lateral direction inside the spot $|y| \leqslant w$ for simplicity.

To solve Eq. (4), we use the symmetry condition at $y=0$, i.e., $\partial n_{h}(0) / \partial y=0$, and the boundary condition for the density flow at the edge of the spot, $n_{h}(w) u_{\mathrm{bal}}=-D \partial n_{h}(w) / \partial y$, assuming electrons outside of the spot move ballistically with $u_{\text {bal }}$. The fast electron density distribution inside the spot $(|y| \leqslant w)$ is then obtained as

$$
n_{h}=\gamma n_{c} \Theta \frac{w^{2}}{L^{2}}\left(1+2 \frac{u_{\mathrm{dif}}}{u_{\mathrm{bal}}}-\frac{y^{2}}{w^{2}}\right),
$$

where $\Theta \equiv 1 /(\sin \bar{\theta} \tan \bar{\theta}) \simeq 0.76$. From Eq. (5), we see more accumulation takes place as $w / L$ increases; this is accomplished by either increasing $w$ or decreasing $L$. Note that $n_{h}$ is limited by the original target density. For 3D situations, we can derive the same dependence of $n_{h}$ on $w / L$ as Eq. (5) by solving the diffusion equation in a $2 \mathrm{D}$ plane surface where the mean step size in the lateral direction is given by $\Delta y / 2^{1 / 2}$. The value of $n_{h}$ for the $3 \mathrm{D}$ case has no significant difference from that for $2 \mathrm{D}$, e.g., $n_{h}$ for $3 \mathrm{D}$ is about $10 \%$ less than that for $2 \mathrm{D}$ for $w / L=7$. The details are given in Appendix A.

The accumulation to the density $n_{h}$ given by Eq. (5) needs enough time longer than $\tau_{\text {conf }} \equiv \int n_{h} d V / \int S d V$, which corresponds to the time for the fast electron density to reach $n_{h}$ without having the diffusion. Here the integral is taken over the volume of the accumulation region. Using Eq. (5), we obtain the confinement time as

$$
\tau_{\text {conf }}=\frac{w}{3 u_{\mathrm{dif}}}\left(1+3 \frac{u_{\mathrm{dif}}}{u_{\mathrm{bal}}}\right) .
$$

The laser pulse duration has to be longer than $\tau_{\text {conf }}$ to obtain the benefit of the lateral confinement, e.g., $\tau_{\text {conf }} \simeq 0.6$ ps for $w=35 \mu \mathrm{m}$ and $L=5 \mu \mathrm{m}$.

During the accumulation, the plasma expands with the sound speed $C_{s}=\left(Z_{i} T_{h} / M_{i}\right)^{1 / 2}$ where $Z_{i}$ is the ion charge state, $M_{i}$ is the ion mass, and $T_{h}$ is the average energy of fast electrons. We here use $T_{h} \simeq T_{p}=(\gamma-1) m_{e} c^{2}$. Due to the expansion, the longitudinal length of the circulation $L$ increases gradually from the initial foil thickness. To estimate $n_{h}$ with the expansion effect, we substitute

$$
L \equiv L_{0}+2 C_{s} \bar{\tau}_{\text {conf }}
$$

to Eq. (5) where $\bar{\tau}_{\text {conf }}$ is the confinement time derived from Eq. (6) with replacing $L$ by $\left(L+L_{0}\right) / 2$ in the calculation of $u_{\text {dif }}$ [Eq. (3)], $L_{0}$ is the initial foil thickness, and we consider the expansion in both front and rear surfaces. By solving Eq. (7) for $L$, we have

$$
\frac{L}{L_{0}}=\left(1+2 C_{0} \chi \frac{w^{2}}{L_{0}^{2}}+2 \chi \frac{w}{L_{0}}+\chi^{2} \frac{w^{2}}{L_{0}^{2}}\right)^{1 / 2}+\chi \frac{w}{L_{0}} .
$$


We use Eq. (8) as the longitudinal circulation length of fast electrons in the density accumulation process. Here $\chi \equiv C_{s} / u_{\text {bal }}$ and $C_{0} \equiv 4 /(3 \tan \bar{\theta}) \simeq 0.86$. Without expansion $C_{s}=0$, we reproduce $L=L_{0}$ from Eq. (8). The factor $\chi \simeq$ $0.02\left(T_{p} / m_{e} c^{2}\right)^{1 / 2} / \beta$ is smaller than unity for the amplitude regime considered here, $a_{0} \lesssim 100$, for fully ionized deuteronlike plasmas where $\beta \equiv v / c$.

When we use an approximation $2 \chi w / L_{0} \ll 1$, i.e., we limit the parameter regime to $w / L_{0}<(2 \chi)^{-1}$ where $(2 \chi)^{-1} \simeq$ $25(\gamma+1)^{1 / 2} / \gamma$, Eq. (8) is reduced to

$$
L \simeq L_{0}\left(1+2 C_{0} \chi \frac{w^{2}}{L_{0}^{2}}\right)^{1 / 2},
$$

where $C_{0} \chi=4 \Theta C_{s} / 3 v \simeq C_{s} / v$ indicates the ratio of the sound velocity to the average fast electron velocity. We substitute Eq. (9) to Eq. (5) and obtain

$$
n_{h}=\gamma n_{c} \Theta \frac{w^{2} / L_{0}^{2}}{1+2 C_{0} \chi w^{2} / L_{0}^{2}}\left(1+2 \frac{u_{\mathrm{dif}}}{u_{\mathrm{bal}}}-\frac{y^{2}}{w^{2}}\right),
$$

which shows the dependence of the accumulation density of fast electrons on the ratio of spot radius $w$ and initial foil thickness $L_{0}$. For small $w / L_{0}\left(2 C_{0} \chi w^{2} / L_{0}^{2} \ll 1\right), n_{h}$ is proportional to $\left(w / L_{0}\right)^{2}$.

In the limit of large spot-thickness ratio where $C_{0} \chi w^{2} / L_{0}^{2} \gg 1$ and $w / L_{0} \gg 1$ are satisfied, $L$ given by Eq. (8) becomes $L \simeq L_{0}\left(2 C_{0} \chi w^{2} / L_{0}^{2}\right)^{1 / 2}$. In this case, the diffusion velocity Eq. (3) is approximated as $u_{\text {dif }} / u_{\text {bal }} \simeq\left(8 \chi / C_{0}\right)^{1 / 2} / 3 \simeq\left(C_{s} / u_{\text {bal }}\right)^{1 / 2}$. The peak fast electron density, $n_{h}$ at $y=0$ in Eq. (5), then approaches

$$
n_{h}(y=0) \simeq \frac{3}{8} \frac{v}{C_{s}} \gamma n_{c}\left(1+\frac{4}{3} \sqrt{\frac{2 \chi}{C_{0}}}\right),
$$

which is independent of $w / L_{0}$. Using $\bar{\theta}=1$ [Eq. (1)], we can rewrite the second term on the right-hand side (r.h.s.) of Eq. (11) approximately as $2 C_{s} / u_{\mathrm{bal}}$, which is negligible compared with the first term. Equation (11) is the upper limit of the fast electron density achieved by the recirculation in the expanding foil plasma. For instance, for $a_{0}=1.4$ and fully ionized deuteron-like plasma, we obtain $n_{h}(y=0) \simeq 43 n_{c}$ from Eq. (11). Note that when the effect of plasma expansion is not included, i.e., if we merely replace $w / L$ by $w / L_{0}$ in Eq. (5), the accumulation density $n_{h}$ becomes infinity as $w / L_{0}$ increases.

We show the fast electron density at the laser axis $(y=0)$ in the foil in the simulations (points) and that obtained from Eq. (10) (background color) in Fig. 5(a). Note that the colored area satisfies the condition $2 \chi w / L_{0}<1$ where Eq. (10) is applicable. The black dashed lines are contour lines for the background color. In the simulations, we averaged the densities in the $x$ direction inside the foil. The threshold energy of the fast electrons is defined as $50 \mathrm{keV}$ multiplied by a factor $\tilde{L}_{0} \gamma / \gamma_{0}$. Here $\gamma_{0}$ is the relativistic factor for the reference case $a_{0}=1.4 . \tilde{L}_{0}$ is the initial foil thickness normalized by the reference thickness $5 \mu \mathrm{m}$ in order to exclude the hot bulk electrons fairly, since the bulk electrons are heated through the anomalous heating in the kinetic simulation [37] during the confinement time $\tau_{\text {conf }}$ which is longer for thicker target. The two shaded areas, ballistic and target disassembly, are ineffi-

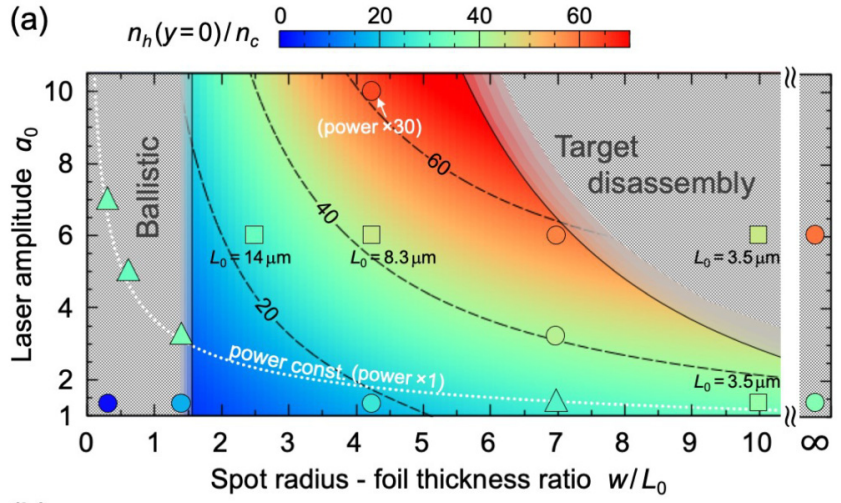

(b)

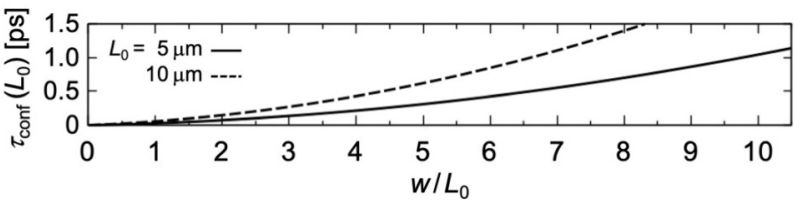

FIG. 5. (a) Fast electron densities at $y=0$ averaged inside the target in the PIC simulations (points) and that derived from the theory Eq. (10), $n_{h}(y=0)$ (background color). The black dashed lines are contour lines for the background color. The shaded areas are the regimes where the diffusion model is not applicable, i.e., the ballistic regime $\Delta y \geqslant w$ where the solid vertical line corresponds to $\Delta y=w$ (left), and the target disassembly regime $\tau_{\text {conf }} \geqslant \tau_{\text {dis }}$ for the initial target density $n_{0}=100 n_{c}$ (right). The initial foil thickness $L_{0}$ in the simulations is $5 \mu \mathrm{m}$ except for the squares. The white dotted line is a contour line for laser power for the same $L_{0}$. At $w / L_{0}=\infty$, simulation results for a quasi-1D condition with a periodic $y$-boundary are shown. The simulation values at $w / L_{0}=\infty$ can be reproduced well by Eq. (11). (b) The confinement time Eq. (6) for $L_{0}=5 \mu \mathrm{m}$ and $10 \mu \mathrm{m}$ with the relativistic approximation $\beta=1$.

cient regimes for the lateral confinement. Namely, for small $w / L_{0}$ where $w \leqslant \Delta y$, the electrons do not show the random walk, and they escape from the spot area almost ballistically. As for large $w / L_{0}$, we consider the limitation by the target disassembly, i.e., the bulk target disassembles before $n_{h}$ reaches the steady-state density. The disassemble time is estimated as $\tau_{\text {dis }}=\left(L_{0} / 2\right) / C_{\text {s cold }}$ where $C_{\text {s cold }}=\left(Z_{i} T_{c} / M_{i}\right)^{1 / 2}$ is the sound speed of the cold bulk plasma, and $T_{c} \equiv\left(\gamma n_{c} / n_{0}\right)^{1 / 2} T_{p}$ is the bulk electron temperature heated by the laser light [38]. The confinement time should be shorter than $\tau_{\text {dis }}$, otherwise one enters the target disassembly regime in Fig. 5(a). Here we assume $n_{0}=100 n_{c}$, and estimate $\tau_{\text {conf }}$ with $L=L_{0}$ for simplicity as plotted in Fig. 5(b). The detailed discussions of the limit for large $w / L_{0}$ and $a_{0}$ are given in Appendix B.

We see from Fig. 5(a) that $n_{h}$ is higher for larger $w / L_{0}$ where the efficient electron accumulation with the random walk occurs, and $n_{h}$ is higher for larger $a_{0}$ due to the increase of the source density $\gamma n_{c}$. However, when we enter the target disassembly regime, $n_{h}$ reduces as seen from the simulation point at $a_{0}=6$ and $w / L_{0}=10$. We also show the simulation results for a quasi-1D condition by setting a periodic boundary with a $4 \mu \mathrm{m}$ simulation box size laterally, imitating $w / L_{0} \rightarrow \infty$. These values indicate the accumulation densities limited only by the target expansion under the continuous 

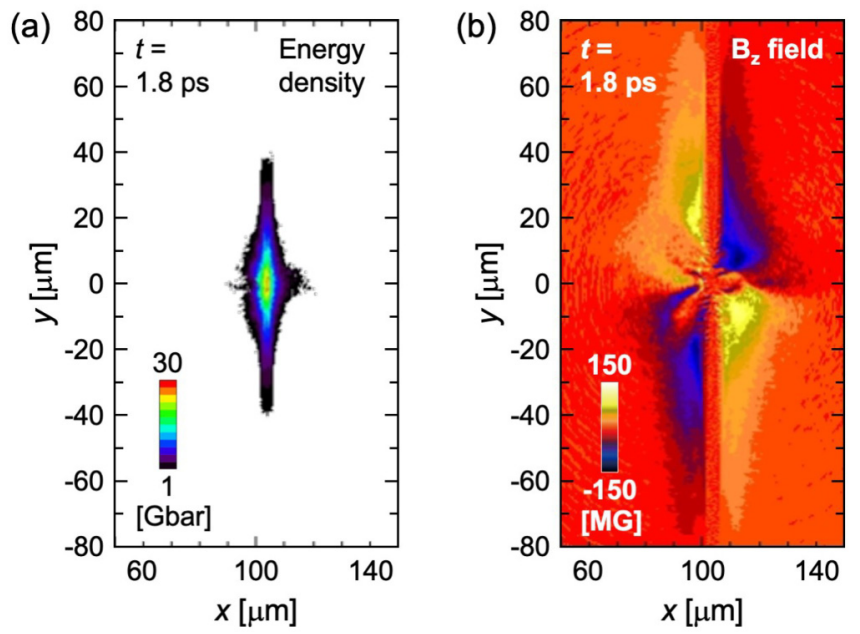

FIG. 6. 2D snapshots of (a) electron energy density and (b) azimuthal magnetic field $B_{z}$ at $t=1.8 \mathrm{ps}$ in the PIC simulation with laser field amplitude $a_{0}=7.0$, spot radius $w=1.4 \mu \mathrm{m}$, and initial foil thickness $L_{0}=5 \mu \mathrm{m}$.

laser irradiation. The simulation values at $w / L_{0}=\infty$ are well described by Eq. (11).

The triangles connected by a white dotted line present the simulations with the same laser power in the 2D geometry, i.e., $a_{0}$ scales with $w^{-1 / 2}$. All of them show similar density values as $(32 \pm 3) n_{c}$. As we increase the input power about 30 times, the fast electron density reaches $\simeq 64 n_{c}$ as shown by the point at $a_{0}=10$ and $w / L_{0}=4.2$.

The optimum laser and target condition to achieve high fast electron densities is located around the boundary of the target disassembly regime. For instance, when we have $1 \mathrm{~kJ}$ laser energy with pulse length $\tau_{\text {pulse }}=1.8 \mathrm{ps}$, we can choose $5 \mu \mathrm{m}$-thick target $\left(L_{0}=5 \mu \mathrm{m}\right)$ and $w / L_{0}=7$ where $\tau_{\text {conf }} \simeq$ 0.6 ps [see Fig. 5 (b) solid line] satisfies $\tau_{\text {conf }} \ll \tau_{\text {pulse. }}$. The average $a_{0}$ is 3.2 for the given laser energy, and we expect $n_{h} \simeq 35 n_{c}$ from Fig. 5(a). If we reduce the foil thickness and spot size to one third keeping $w / L_{0}=7$, the amplitude can be $a_{0}=9.8$ with the same laser energy, but we enter the disassembly regime and do not expect the full benefit of the confinement considered here. Note that the target must be thicker than the skin depth of the bulk plasma. In Fig. 5(b) we use approximations $\beta=1$ and $L=L_{0}$.

Inside the ballistic regime, the simulations show higher $n_{h}$ than the source density $\gamma n_{c}$ especially for higher $a_{0}$. This is due to the global magnetic field which appears at the edge of the spot. Figures 6(a) and 6(b) show the electron density and the azimuthal magnetic field, respectively, in the small spot case with $a_{0}=7.0, w=1.4 \mu \mathrm{m}$, and $L_{0}=5 \mu \mathrm{m}$. The global magnetic field surrounding the laser spot area grows to approximately $150 \mathrm{MG}$, which is about $20 \%$ of the magnetic field strength of the incident laser light $\left(a_{0}=7.0\right)$. The corresponding magnetic field pressure is $\sim$ Gbar, which is comparable to the electron energy density at the peripheral of the spot as seen in Fig. 6(a). The Larmor radius of electrons with energy $0.5 \mathrm{MeV}$ in the magnetic field of $150 \mathrm{MG}$ is about $1.4 \mu \mathrm{m}$, i.e., the same as the spot radius. Hence, the global magnetic field seen in Fig. 6(b) confines the electron com-
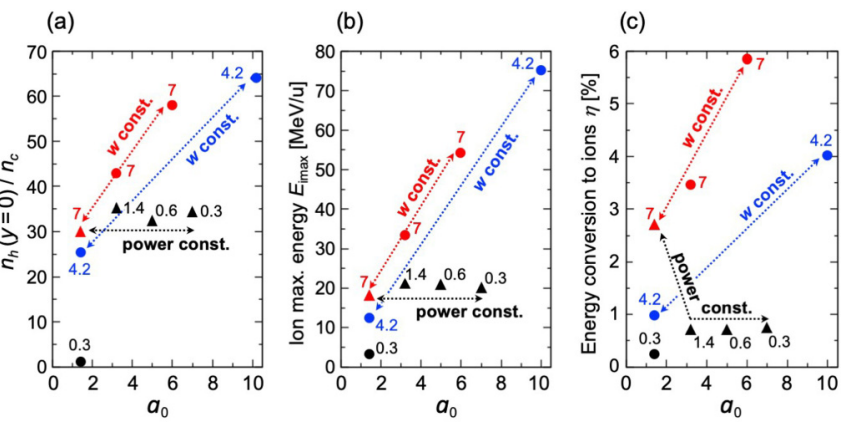

FIG. 7. (a) Peak fast electron density, (b) maximum energy of deuteron ions from the target rear $E_{\text {imax }}$, and (c) energy conversion efficiency $\eta$ from laser to ions with energies over $1 \mathrm{MeV} / \mathrm{u}$ and divergence angle $\pm 0.1 \mathrm{rad}$ from the target rear in the simulations. $E_{\text {imax }}$ and $\eta$ are observed when the ion front expands $100 \mu \mathrm{m}$ from the target rear. Spot radii $w$ divided by $L_{0}=5 \mu \mathrm{m}$ are indicated in the plots. The black points are the cases where the diffusion model is not applicable $(w \leqslant \Delta y)$. The triangles present simulations with the same laser power.

ponent below $0.5 \mathrm{MeV}$ which forms a bump in the electron energy spectrum of Fig. 2(c) (blue dashed line).

\section{IMPACT ON ION ACCELERATION}

As an impact of the lateral confinement, we show the ion acceleration properties in the simulations. Here we vary the spot radius $w$ while fix the foil thickness $L_{0}=5 \mu \mathrm{m}$. The value of $w / L_{0}$ is indicated for each point in Fig. 7. In Fig. 7(a) the accumulated fast electron densities $n_{h}(y=0)$ are plotted with respect to $a_{0}$. The dotted arrows are eye guides to follow the cases with the same $w$ and the same laser power. When the spot radius $w$ is constant, $n_{h}$ increases nearly proportional to $a_{0}$, reflecting the increment of the source density by $\gamma \sim a_{0}$. For a constant laser power in the 2D geometry (triangles), $n_{h}$ show little variation for different $a_{0}$, indicating the efficient confinement with larger $w$. Note that the black points present the ballistic cases $w / L_{0}<1.5$. In Fig. 7(b) the maximum energies of ions $E_{\operatorname{imax}}$ accelerated to the positive $x$ direction from the target rear are plotted. $E_{\text {imax }}$ increases nearly proportionally with $a_{0}$ for a fixed $w$ as predicted by the TNSA theory [39]. For a constant laser power, $E_{\text {imax }}$ tends to be the same even for higher $a_{0}$. This denotes that tight focusing does not benefit the ion acceleration [40].

We see Figs. 7(a) $n_{h}$ and 7(b) $E_{\text {imax }}$ show similar dependencies on $a_{0}$, which indicates the confinement of fast electrons is essential for the TNSA ion acceleration. Namely, the confinement increases the density of fast electrons as well as their average energy beyond the ponderomotive scaling. Since the ion maximum energy $E_{\text {imax }}$ depends on the time integral of the electron average energy, the temporal increase of the electron energy impacts on the ion maximum energy [25], while the accumulation of the fast electrons in the recirculation also contributes to boost the ion maximum energy $[15,16]$. Moreover, as seen in Fig. 7(c), large spot lasers have an advantage in regard to the energy conversion efficiency $\eta$ from laser to ions. Here $\eta$ is evaluated for ions at the rear side with energies over $\mathrm{MeV}$ per nucleon $(\mathrm{MeV} / \mathrm{u})$ and divergence within $\pm 0.1 \mathrm{rad}$ 
as a preferable ion source for the applications. By comparing the constant power cases, we see $\eta$ significantly higher in the diffusion regime (red triangle) than those in the ballistic regime (black triangles). The increase of $\eta$ with $a_{0}$ is higher for larger $w / L_{0}$, as seen from the gradient of the red and blue arrows. Other ion acceleration mechanisms such as hole boring and relativistic transparency will appear for a higher $a_{0}$ regime where the lateral confinement effect shown here is weak as discussed in Appendix B.

\section{CONCLUSION}

In conclusion, using PIC simulations for relativistic laserthin foil interactions, we demonstrated the fast electrons circulating around the foil show a random walk behavior in the lateral direction via multiple scattering by fluctuating fields at the modulated surfaces of the foil plasma. This diffusive feature appears in interactions with lasers having spot radii $w$ much wider than the foil thickness $L$. Owing to the random walk motion, the average lateral velocity of the fast electrons reduces in proportion to $L / w$ from that for the ballistic motion. Fast electrons accumulate in the spot area, which results in typically 10 times higher density than the source density. This is a lateral confinement of the fast electrons appearing naturally in the large spot laser-foil interaction in over-ps timescale. The confinement makes a quasi-1D situation with small loss of laser energy to the lateral direction. This is a great advantage of large spot lasers and is a key to explaining the superthermal electron generation and efficient ion acceleration seen in experiments using $\mathrm{kJ}$ class lasers such as LFEX [23] and NIF-ARC [24]. Moreover, it will be an efficient way to create high-energy density plasmas for bright $\mathrm{X}$-ray and neutron sources.

\section{ACKNOWLEDGMENTS}

This study was supported by JSPS KAKENHI Grants No. JP20K14439, No. JP20H00140, and No. JP19KK0072, the Foundation of Kinoshita Memorial Enterprise, the Foundation for the Promotion of Ion Engineering, and the DOE Office of Science Early Career Program under Grant No. SCW1265-1. Work was in part performed under the auspices of the US Department of Energy by the Lawrence Livermore National Laboratory under Contract No. DE-AC52-07NA27344 and also under tracking code 17-ERD-039 of the Laboratory Directed Research and Development program.

\section{APPENDIX A: ACCUMULATION DENSITY FOR 3D GEOMETRY}

For the laser-foil interaction in 3D geometry, we consider the diffusion of fast electrons in the 2D plane along the foil surface. We here consider the diffusion in the radial $r$ direction assuming the azimuthal symmetry, and solve the stationary state equation given by

$$
0=D_{r} \nabla^{2} n_{h}(r)+S,
$$

where $D_{r}=(\Delta r)^{2} /(2 \Delta t)$ is the diffusion coefficient in the lateral direction in the 2D plane, and $S=\gamma n_{c} v / L$ is the source term. Here we use the average step size $\Delta r=\Delta y / 2^{1 / 2}$ where
$\Delta y=L \tan \bar{\theta}$ is the average step size in the $2 \mathrm{D}$ geometry, and the average period of the circulation $\Delta t=L /(v \cos \bar{\theta})$, which is the same as that in the $2 \mathrm{D}$ geometry. The diffusion coefficient $D_{r}$ is then given as

$$
D_{r}=\frac{L v}{4 \Theta},
$$

where $\Theta \equiv 1 /(\sin \bar{\theta} \tan \bar{\theta}) \simeq 0.76$ as the main text. The diffusion coefficient Eq. (A2) is half of that in the 2D geometry, $D=(\Delta y)^{2} /(2 \Delta t)=L v /(2 \Theta)$. Using the symmetry condition $\partial n_{h} / \partial r=0$ at $r=0$ and the boundary condition $n_{h} u_{\text {bal }}=$ $-D_{r} \partial n_{h} / \partial r$ at $r=w$, we obtain the accumulation density in the $3 \mathrm{D}$ geometry as

$$
n_{h}^{(3 \mathrm{D})}=\gamma n_{c} \Theta \frac{w^{2}}{L^{2}}\left(1+\frac{\tan \bar{\theta}}{2 w / L}-\frac{r^{2}}{w^{2}}\right) .
$$

The accumulation density in the $2 \mathrm{D}$ geometry Eq. (5) can be rewritten as

$$
n_{h}=\gamma n_{c} \Theta \frac{w^{2}}{L^{2}}\left(1+\frac{\tan \bar{\theta}}{w / L}-\frac{y^{2}}{w^{2}}\right) .
$$

Comparing Eqs. (A3) and (A4), we see that the difference appears only in the second term of the r.h.s. of Eq. (A4). For $w / L=\tan \bar{\theta}$, which is the lower limit of $w / L$ for the diffusion model to be applicable, the accumulation density at the center $r=0$ in the $3 \mathrm{D}$ case Eq. (A3) is $25 \%$ less than that in the $2 \mathrm{D}$ case Eq. (A4), and the difference between Eqs. (A3) and (A4) becomes smaller for larger $w / L$.

\section{APPENDIX B: APPLICABLE REGIME OF THE DIFFUSION MODEL}

We here summarize the conditions for the present diffusion model to be applicable. For small $w / L$, the limit is given by $w / L=\tan \theta$ below which we enter the ballistic regime shown in Fig. 5(a). In the following, we consider the limit for large $w / L$ and $a_{0}$ region.

The target foil has to be sustained during the confinement time Eq. (6). When the bulk plasma expands with the sound speed $C_{\text {scold }}=\left(Z_{i} T_{c} / M_{i}\right)^{1 / 2}$, the foil with initial thickness $L_{0}$ is disassembled in time $\tau_{\text {dis }}$ given by

$$
\frac{L_{0}}{2}=\int_{0}^{\tau_{\mathrm{dis}}} C_{\mathrm{s} \text { cold }} d t .
$$

Here $Z_{i}$ is the ion charge state, $M_{i}$ is the ion mass, and $T_{c}$ is the bulk electron temperature. We have $1 / 2$ on the left-hand side of Eq. (B1) because the plasma expands to both front and rear sides of the foil. We here use $T_{c}=\left(\gamma n_{c} / n_{0}\right)^{1 / 2} T_{p}$ [38], which is constant in time where $T_{p}$ is the ponderomotive energy, $\gamma$ is the average relativistic factor of fast electrons given by the ponderomotive scaling, and $n_{0}$ is the bulk electron density. Equation (B1) then leads to $\tau_{\mathrm{dis}}=L_{0} / 2 C_{\mathrm{s} \text { cold }}$. The confinement time Eq. (6) has to be shorter than $\tau_{\text {dis }}$ as

$$
\tau_{\text {conf }}<\frac{L_{0}}{2 C_{\text {s cold }}} .
$$


The threshold condition $\tau_{\text {conf }}=\tau_{\text {dis }}$ is shown by the black solid line as the edge of the target disassembly regime in Fig. 5(a).

The laser pushes the front surface of the foil by the hole boring with the velocity $v_{\mathrm{HB}}$, and penetrates through the foil eventually. Here $v_{\mathrm{HB}}=c\left(I Z_{i} / n_{0} M_{i} c^{3}\right)^{1 / 2}$ is derived from the momentum transfer equation from a fully reflected laser to ions at the interface [22]. The penetration time $\tau_{\mathrm{HB}}=L_{0} / v_{\mathrm{HB}}$ has to be longer than the confinement time, which is expressed as

$$
\tau_{\text {conf }}<\frac{L_{0}}{v_{\mathrm{HB}}} .
$$

The ratio of $C_{\mathrm{s} \text { cold }}$ and $v_{\mathrm{HB}}$ is given as

$$
\frac{v_{\mathrm{HB}}}{C_{\mathrm{s} \text { cold }}}=\left[\frac{a_{0}^{2}}{2 \gamma^{1 / 2}(\gamma-1)} \frac{Z_{i}}{\left(n_{0} / n_{c}\right)^{1 / 2}}\right]^{1 / 2} .
$$

For large amplitude $a_{0} \gg 1$, we use $\gamma-1 \simeq \gamma \simeq a_{0} / 2^{1 / 2}$ and obtain

$$
\frac{v_{\mathrm{HB}}}{C_{\mathrm{s} \mathrm{cold}}}=\left(\frac{2 a_{0} Z_{i}^{2}}{n_{0} / n_{c}}\right)^{1 / 4},
$$

from which we see $2 C_{\mathrm{s} \text { cold }}>v_{\mathrm{HB}}$ for $a_{0}<8 n_{0} /\left(Z_{i} n_{c}\right)$. Since the the laser amplitude considered in this paper is within the regime $a_{0}<8 n_{0} /\left(Z_{i} n_{c}\right)$, Eq. (B3) is satisfied as long as Eq. (B2) is satisfied. Hence, we plot only the limit given by Eq. (B2) in Fig. 5(a).

Another limit is given by $L_{0}>\ell_{\text {skin }}$, i.e., the foil thickness $L_{0}$ has to be longer than the skin depth at the bulk density, $\ell_{\text {skin }}=\left(n_{c} / n_{0}\right)^{1 / 2} \lambda_{L} / 2 \pi$. Although Eq. (10) indicates that one can obtain higher fast electron density $n_{h}$ by reducing the target thickness $L_{0}$, the foil has to be enough thick so that the laser light does not penetrate through the foil by the relativistic transparency.
[1] A. Macchi, M. Borghesi, and M. Passoni, Ion acceleration by superintense laser-plasma interaction, Rev. Mod. Phys. 85, 751 (2013).

[2] H. Daido, M. Nishiuchi, and A. S. Pirozhkov, Review of laserdriven ion sources and their applications, Rep. Prog. Phys. 75, 056401 (2012).

[3] N. D. Powers, I. Ghebregziabher, G. Golovin, C. Liu, S. Chen, S. Banerjee, J. Zhang, and D. P. Umstadter, Quasimonoenergetic and tunable X-rays from a laser-driven Compton light source, Nat. Photonics 8, 28 (2014).

[4] K. L. Lancaster, S. Karsch, H. Habara, F. N. Beg, E. L. Clark, R. Freeman, M. H. Key, J. A. King, R. Kodama, K. Krushelnick et al., Characterization of ${ }^{7} \mathrm{Li}(\mathrm{p}, \mathrm{n})^{7} \mathrm{Be}$ neutron yields from laser produced ion beams for fast neutron radiography, Phys. Plasmas 11, 3404 (2004).

[5] D. P. Higginson, J. M. McNaney, D. C. Swift, T. Bartal, D. S. Hey, R. Kodama, S. Le Pape, A. Mackinnon, D. Mariscal, H. Nakamura et al., Laser generated neutron source for neutron resonance spectroscopy, Phys. Plasmas 17, 100701 (2010).

[6] O. Komeda, Y. Nishimura, Y. Mori, R. Hanayama, K. Ishii, S. Nakayama, Y. Kitagawa, T. Sekine, N. Sato, T. Kurita et al., First demonstration of laser engagement of 1-Hz-injected flying pellets and neutron generation, Sci. Rep. 3, 2561 (2013).

[7] H. Chen, F. Fiuza, A. Link, A. Hazi, M. Hill, D. Hoarty, S. James, S. Kerr, D. D. Meyerhofer, J. Myatt et al., Scaling the Yield of Laser-Driven Electron-Positron Jets to Laboratory Astrophysical Applications, Phys. Rev. Lett. 114, 215001 (2015).

[8] G. Sarri, K. Poder, J. M. Cole, W. Schumaker, A. Di Piazza, B. Reville, T. Dzelzainis, D. Doria, L. A. Gizzi, G. Grittani et al., Generation of neutral and high-density electron-positron pair plasmas in the laboratory, Nat. Commun. 6, 6747 (2015).

[9] M. Tabak, J. Hammer, M. E. Glinsky, W. L. Kruer, S. C. Wilks, J. Woodworth, E. M. Campbell, and M. D. Perry, Ignition and high gain with ultrapowerful lasers, Phys. Plasmas 1, 1626 (1994).

[10] R. Kodama, P. A. Norreys, K. Mima, A. E. Dangor, R. G. Evans, H. Fujita, Y. Kitagawa, K. Krushelnick, T. Miyakoshi, N. Miyanaga et al., Fast heating of ultrahigh-density plasma as a step towards laser fusion ignition, Nature (London) 412, 798 (2001).

[11] S. Sakata, S. Lee, H. Morita, T. Johzaki, H. Sawada, Y. Iwasa, K. Matsuo, K. F. F. Law, A. Yao, M. Hata et al., Magnetized fast isochoric laser heating for efficient creation of ultra-highenergy-density states, Nat. Commun. 9, 3937 (2018).

[12] H. Sawada, Y. Sentoku, T. Yabuuchi, U. Zastrau, E. Forster, F. N. Beg, H. Chen, A. J. Kemp, H. S. McLean, P. K. Patel, and Y. Ping, Monochromatic 2D $K \alpha$ Emission Images Revealing Short-Pulse Laser Isochoric Heating Mechanism, Phys. Rev. Lett. 122, 155002 (2019).

[13] A. P. L. Robinson, H. Schmitz, J. S. Green, C. P. Ridgers, and N. Booth, Guiding of laser-generated fast electrons by exploiting the resistivity-gradients around a conical guide element, Plasma Phys. Controlled Fusion 57, 064004 (2015).

[14] C. P. Ridgers, M. Sherlock, R. G. Evans, A. P. L. Robinson, and R. J. Kingham, Superluminal sheath-field expansion and fast-electron-beam divergence measurements in laser-solid interactions, Phys. Rev. E 83, 036404 (2011).

[15] A. J. Mackinnon, Y. Sentoku, P. K. Patel, D. W. Price, S. Hatchett, M. H. Key, C. Andersen, R. Snavely, and R. R. Freeman, Enhancement of Proton Acceleration by Hot-Electron Recirculation in Thin Foils Irradiated by Ultraintense Laser Pulses, Phys. Rev. Lett. 88, 215006 (2002).

[16] Y. Sentoku, T. E. Cowan, A. Kemp, and H. Ruhl, High energy proton acceleration in interaction of short laser pulse with dense plasma target, Phys. Plasmas 10, 2009 (2003).

[17] R. J. Gray, R. Wilson, M. King, S. D. R. Williamson, R. J. Dance, C. Armstrong, C. Brabetz, F. Wagner, B. Zielbauer, V. Bagnoud et al., Enhanced laser-energy coupling to dense plasmas driven by recirculating electron currents, New J. Phys. 20, 033021 (2018).

[18] N. Miyanaga, H. Azechi, K. A. Tanaka, T. Kanabe, T. Jitsuno, J. Kawanaka, Y. Fujimoto, R. Kodama, H. Shiraga, K. Knodo et al., 10-kJ PW laser for the FIREX-I program, J. Phys. IV (France) 133, 81 (2006).

[19] J. K. Crane, G. Tietbohl, P. Arnold, E. S. Bliss, C. Boley, G. Britten, G. Brunton, W. Clark, J. W. Dawson, S. Fochs et al., 
Progress on converting a NIF quad to eight, petawatt beams for advanced radiography, J. Phys.: Conf. Ser. 244, 032003 (2010).

[20] D. N. Maywar, J. H. Kelly, L. J. Waxer, S. F. B. Morse, I. A. Begishev, J. Bromage, C. Dorrer, J. L. Edwards, L. Folnsbee, M. J. Guardalben et al., OMEGA EP high-energy petawatt laser: Progress and prospects, J. Phys.: Conf. Ser. 112, 032007 (2008).

[21] D. Batani, M. Koenig, J. L. Miquel, J. E. Ducret, E. dHumieres, S. Hulin, J. Caron, J. L. Feugeas, Ph. Nicolai, V. Tikhonchuk et al., Development of the PETawatt Aquitaine Laser system and new perspectives in physics, Phys. Scr. 2014, 014016 (2014).

[22] S. C. Wilks, W. L. Kruer, M. Tabak, and A. B. Langdon, Absorption of Ultra-Intense Laser Pulses, Phys. Rev. Lett. 69, 1383 (1992).

[23] A. Yogo, K. Mima, N. Iwata, S. Tosaki, A. Morace, Y. Arikawa, S. Fujioka, T. Johzaki, Y. Sentoku, H. Nishimura et al., Boosting laser-ion acceleration with multi-picosecond pulses, Sci. Rep. 7, 42451 (2017).

[24] D. Mariscal, T. Ma, S. C. Wilks, A. J. Kemp, G. J. Williams, P. Michel, H. Chen, P. K. Patel, B. A. Remington, M. Bowers et al., First demonstration of ARC-accelerated proton beams at the National Ignition Facility, Phys. Plasmas 26, 043110 (2019).

[25] N. Iwata, K. Mima, Y. Sentoku, A. Yogo, H. Nagatomo, H. Nishimura, and H. Azechi, Fast ion acceleration in a foil plasma heated by a multi-picosecond high intensity laser, Phys. Plasmas 24, 073111 (2017).

[26] J. Kim, A. J. Kemp, S. C. Wilks, D. H. Kalantar, S. Kerr, D. Mariscal, F. N. Beg, C. McGuffey, and T. Ma, Computational modeling of proton acceleration with multi-picosecond and high energy, kilojoule, lasers, Phys. Plasmas 25, 083109 (2018).

[27] N. Iwata, Y. Sentoku, T. Sano, and K. Mima, Plasma expansion accompanying superthermal electrons in over-picosecond relativistic laser-foil interactions, Plasma Phys. Controlled Fusion 62, 014011 (2020).

[28] N. Iwata, Y. Sentoku, T. Sano, and K. Mima, Electron acceleration in dense plasmas heated by a picosecond relativistic laser, Nucl. Fusion 59, 086035 (2019).
[29] A. J. Kemp and L. Divol, Interaction Physics of Multipicosecond Petawatt Laser Pulses with Overdense Plasma, Phys. Rev. Lett. 109, 195005 (2012).

[30] A. Sorokovikova, A. V. Arefiev, C. McGuffey, B. Qiao, A. P. L. Robinson, M. S. Wei, H. S. McLean, and F. N. Beg, Generation of Superponderomotive Electrons in Multipicosecond Interactions of Kilojoule Laser Beams with Solid-Density Plasmas, Phys. Rev. Lett. 116, 155001 (2016).

[31] N. Iwata, S. Kojima, Y. Sentoku, M. Hata, and K. Mima, Plasma density limits for hole boring by intense laser pulses, Nat. Commun. 9, 623 (2018).

[32] Y. Sentoku and A. J. Kemp, Numerical methods for particle simulations at extreme densities and temperatures: Weighted particles, relativistic collisions and reduced currents, J. Comput. Phys. 227, 6846 (2008).

[33] E. S. Weibel, Spontaneously Growing Transverse Waves in a Plasma Due to an Anisotropic Velocity Distribution, Phys. Rev. Lett. 2, 83 (1959).

[34] Y. Sentoku, K. Mima, S. Kojima, and H. Ruhl, Magnetic instability by the relativistic laser pulses in overdense plasmas, Phys. Plasmas 7, 689 (2000).

[35] A. Macchi, F. Cornolti, F. Pegoraro, T. V. Liseikina, H. Ruhl, and V. A. Vshivkov, Surface Oscillations in Overdense Plasmas Irradiated by Ultrashort Laser Pulses, Phys. Rev. Lett. 87, 205004 (2001).

[36] P. Degond, Transport of trapped particles in a surface potential, Stud. Math. Appl. 31, 273 (2002).

[37] Y. Sentoku, K. Mima, P. Kaw, and K. Nishihara, Anomalous Resistivity Resulting from MeV-Electron Transport in Overdense Plasma, Phys. Rev. Lett. 90, 155001 (2003).

[38] B. Chrisman, Y. Sentoku, and A. J. Kemp, Intensity scaling of hot electron energy coupling in cone-guided fast ignition, Phys. Plasmas 15, 056309 (2008).

[39] P. Mora, Plasma Expansion into a Vacuum, Phys. Rev. Lett. 90, 185002 (2003).

[40] M. Nakatsutsumi, Y. Sentoku, A. Korzhimanov, S. N. Chen, S. Buffechoux, A. Kon, B. Atherton, P. Audebert, M. Geissel, L. Hurd et al., Self-generated surface magnetic fields inhibit laser-driven sheath acceleration of high-energy protons, Nat. Commun. 9, 280 (2018). 